\title{
Secretory activity of subcutaneous abdominal adipose tissue in male patients with rheumatoid arthritis and osteoarthritis - association with clinical and laboratory data
}

\author{
Ewa Kontny ${ }^{1}$, Agnieszka Zielińska², Krystyna-Księżopolska-Orłowska ${ }^{3}$, Piotr Głuszko ${ }^{2}$ \\ ${ }^{1}$ Department of Pathophysiology and Immunology, National Institute of Geriatrics, Rheumatology and Rehabilitation, Warsaw, Poland \\ 2Department of Rheumatology, National Institute of Geriatrics, Rheumatology and Rehabilitation, Warsaw, Poland \\ ${ }^{3}$ Department of Rehabilitation in Rheumatology, National Institute of Geriatrics, Rheumatology and Rehabilitation, Warsaw, Poland
}

\begin{abstract}
Introduction: Adipose tissue exerts widespread effects on the metabolism and immune system, but its activity differs between the genders. In the general population low-grade adipose tissue inflammation contributes to development of diseases of affluence. Little is known about the systemic impact of peripheral fat tissue in osteoarthritis (OA) and rheumatoid arthritis (RA), characterized by chronic, low- and high-grade systemic inflammation, respectively. To clarify this we evaluated the secretory activity of subcutaneous abdominal adipose tissue (SAAT) obtained from male patients affected with RA $(n=21)$ and OA $(n=13)$, and assessed its association with body mass and composition, demographic, clinical and laboratory data.

Material and methods: Basal and interleukin (IL)-1 $\beta$-triggered secretion of selected adipocytokines from SAAT explants was measured by specific enzyme-linked immunosorbent assays (ELISA). Patients' body composition was evaluated by bioelectric impendence technique.

Results: Rheumatoid SAAT secreted more adiponectin and macrophage migration inhibitory factor (MIF) than respective osteoarthritis tissue. In both RA and OA patient groups, stimulation of SAAT explants with IL-1 13 ng/ml/100 mg tissue) significantly up-regulated release of pro-(IL-6, IL-8, tumor necrosis factor - TNF) and anti-inflammatory (IL-10) cytokines but had no effect on the secretion of adiponectin, leptin, MIF and hepatocyte growth factor (HGF). Compared with RA, patients with OA were more obese. In RA patients SAAT-released adiponectin and TNF inversely correlated with body mass index (BMI) and visceral fat rating (FVSC). In addition, SAAT-secreted adiponectin and leptin positively correlated with DAS28 and disease duration, respectively. In the OA group tissue-released TNF positively correlated with patients' age.

Conclusions: We conclude that in RA male patients adipocytokines originating from SAAT are of clinical importance because: (i) adiponectin and TNF may contribute to maintenance of normal body composition and mass, (ii) in addition adiponectin may play a pathogenic role. Moreover, in both RA and OA male patients secretory activity of SAAT may vary with time.
\end{abstract}

Key words: osteoarthritis, rheumatoid arthritis, men, adipose tissue activity.

\section{Introduction}

Rheumatoid arthritis (RA), an autoimmune disease leading to irreversible erosive joint destruction, and osteoarthritis (OA), a chronic musculoskeletal disorder characterized primarily by degradation of articular cartilage, are accompanied by persistent high-grade and low-grade systemic inflammation, respectively [1-5]. Chronic inflammation is thought to contribute to a high 
rate of comorbidity, characteristic for both RA and OA $[4,6]$. Moreover, RA and OA patients have often altered body composition. Obesity, frequent in OA, is also an important risk factor for disease development [7]. By contrast, the majority of RA patients have reduced lean tissue, preserved or increase fat mass along with stable or increased body weight. This condition, named "rheumatoid cachexia", is thought to exert an impact on morbidity and mortality, while obesity seems to be associated with higher RA severity, comorbidities and limited response to treatment, but it may mitigate joint damage $[8,9]$. Therefore, adipose tissue, a dynamic and metabolically active organ which exerts widespread effects on the metabolism, immune function, and vascular homeostasis, is now recognized as a potential contributor to both RA and OA pathogenesis [10]. Fat depots produce multiple soluble factors, named adipocytokines, that mediate numerous adipose tissue biological activities. We have previously found that adipocytokines released from intra-articular fat are involved in RA knee joint pathology, and others have shown a similar role of this tissue in OA [11, 12]. It is known that fat depots from different body locations exhibit distinct features and functional characteristics [13]. In addition, secretory activity of adipose tissue is changed upon exposure to inflammatory stimuli [11]. Thus far, little is known about the systemic impact of peripheral fat tissue in rheumatic diseases distinguished by the intensity of the systemic inflammatory response, i.e. RA and OA. We have recently reported significant differences in secretory activity of subcutaneous abdominal adipose tissue (SAAT) and its different clinical impact in rheumatoid arthritis versus osteoarthritis in female patients [14]. It is worth pointing out that RA affects mostly women while in OA female gender predominates among older patients and in the cases of more severe form of illness. Because there is sexual dimorphism in adipose tissue function, it is hard to predict whether such dissimilarities also discriminate men suffering from these diseases [15].

To clarify this, in the present study we evaluated basal and stimulated secretory activity of SAAT obtained from male patients suffering from RA or OA and assessed its association with body composition, demographic, clinical and laboratory data.

\section{Material and methods}

This study included 21 men who fulfilled the American College of Rheumatology (ACR) 1987 criteria for RA [16] and 13 men with OA diagnosed on the basis of X-ray and clinical judgment. The majority of OA patients had spine and knee or hip involvement. Patients' characteristics are summarized in Table I. Specimens of subcutaneous abdominal fat were taken by $18 \mathrm{G}$ needle biopsy from patients screened for amyloidosis, as previously described [14]. Body composition of patients was evaluated by measurement of total fat mass (tFM), fat-free mass (FFM), and visceral fat $\left(F_{\mathrm{vsC}}\right)$ rating by bioelectric impendence technique using the body composition analyzer Tanita BC-418 MA (Tanita Corp., Tokyo, Japan). Body mass index (BMI) was also calculated. The study meets all criteria contained in the Declaration of Helsinki and was approved by the Ethics Committee of the Institute of Geriatrics, Rheumatology, and Rehabilitation, Warsaw, Poland. All patients provided written informed consent prior to enrolment.

Preparation and in vitro cultures of tissue explants were performed as described previously [11]. Briefly, tissue explants $(100 \mathrm{mg} / \mathrm{ml})$ were pre-cultured for quiescence $\left(26 \mathrm{~h}, 37^{\circ} \mathrm{C}, 5 \% \mathrm{CO}_{2}\right)$ in Dulbecco's modified Eagle medium supplemented with antibiotics (50 $\mathrm{mg}$ of gentamycin, $100 \mathrm{mg} / \mathrm{ml}$ kanamycin). Then the culture medium was replaced with a fresh one and tissue explants were either stimulated for $18 \mathrm{~h}$ with $1 \mathrm{ng} / \mathrm{ml}$ of recombinant human interleukin (IL)-1 $\beta$ (IL-1 $\beta$; R\&D Systems, Minneapolis, MN, USA) or were left untreated (control cultures). To evaluate basal (control cultures) and IL-1 $\beta$-triggered secretory activity of SAAT, the concentrations of selected factors were measured in culture supernatants by enzyme-linked immunosorbent assays (ELISAs). The ELISAs for IL-6 and IL-8 were performed as previously described [11]. The other tests were done using commercially available ELISA sets: the Duo Sets from R\&D Systems for macrophage migration inhibitory factor (MIF), hepatocyte growth factor (HGF), leptin and adiponectin and the Ready-Set-Go sets from eBioscience (San Diego, CA, USA) for tumor necrosis factor (TNF) and IL-10.

Statistical analysis was performed using Statistica 10 software (Stat Soft Inc., Tulsa, OK, USA). The MannWhitney $U$ test or Fisher's two-tailed exact test was used for intergroup (RA vs. OA) comparison of continuous or discrete variables, respectively. The Wilcoxon signedrank test was applied to evaluate the effect of IL-1 $\beta$ stimulation of tissue explants obtained from the same patients (paired samples). Correlation was assessed using Spearman's rank two-tailed test ( $R$ value is shown). $P$ values $<0.05$ were considered significant.

\section{Results}

Baseline characteristics of the patients are shown in Table I. The group of RA and OA patients did not differ significantly in the patients' age, cardiovascular disease (CVD) or diabetes frequency. In both groups the majority of patients suffered from arterial hypertension and myocardial infarctions occurred only in RA. Amyloidosis was rare and occurred only in $5 \%$ of men with RA. Non-ste- 
Table I. Baseline characteristics of patients. Except where indicated otherwise, values are the median (interquartile range)

\begin{tabular}{|c|c|c|c|}
\hline Parametr & $\mathrm{RA}(n=21)$ & $\mathrm{OA}(n=13)$ & $p$ value \\
\hline \multicolumn{4}{|l|}{ Demographics } \\
\hline Age, years & $56(51-61)$ & $61(56-68)$ & 0.22 \\
\hline Disease duration, months & $78(24-204)$ & $108(96-120)$ & \\
\hline \multicolumn{4}{|l|}{ Body composition } \\
\hline BMI & $25.3(22.3-27.2)$ & $30.2(25-33)$ & 0.009 \\
\hline $\mathrm{FM}, \mathrm{kg}$ & $15.1(10.9-20.4)$ & $23.9(14.9-30.3)$ & 0.008 \\
\hline FFM, kg & $58.2(54.9-66.1)$ & $67(62.4-70.6)$ & 0.029 \\
\hline$F_{\text {vSC }}$ & $7.5(5-10)$ & $15(12-16)$ & $<0.0001$ \\
\hline Disease activity (DAS28) & $5.52(4.3-6.4)$ & & \\
\hline \multicolumn{4}{|l|}{ Laboratory values } \\
\hline $\mathrm{ESR}, \mathrm{mm} / \mathrm{h}$ & $31(23-55)$ & $16.0(8-21)$ & 0.003 \\
\hline CRP, mg/l & $23(15-38)$ & $8(6-9)$ & $<0.0001$ \\
\hline Total cholesterol, mg/dl & $198(174-222.5)$ & $171(144-183)$ & 0.019 \\
\hline HDL cholesterol, mg/dl & $40(30-49)$ & $44(38-56)$ & 0.62 \\
\hline LDL cholesterol, mg/dl & $120(85-138)$ & $82(74-109)$ & 0.028 \\
\hline Triglycerides, g/dl & $141(120-247)$ & $156(97-192)$ & 1.0 \\
\hline Atherogenic index & $4.8(3.8-6.1)$ & $3.9(3.2-4.7)$ & 0.14 \\
\hline \multicolumn{4}{|l|}{ Serum cytokine concentrations } \\
\hline $\mathrm{IL}-6, \mathrm{pg} / \mathrm{ml}$ & $180(46-654)$ & $13(0-55)$ & 0.035 \\
\hline IL-8, pg/ml & $2.0(0-105)$ & $0(0-25.6)$ & 0.65 \\
\hline TNF, pg/ml & $0(0-2.0)$ & $0(0-0)$ & 0.80 \\
\hline $\mathrm{MIF}, \mathrm{pg} / \mathrm{ml}$ & $474(268-621)$ & $97(73-106)$ & $<0.0001$ \\
\hline HGF, pg/ml & $481(400-544)$ & $571(453-795)$ & 0.13 \\
\hline Adiponectin, $\mu \mathrm{g} / \mathrm{ml}$ & $5.3(3.9-7.4)$ & $4.9(3.7-6.9)$ & 0.53 \\
\hline Leptin, ng/ml & $7.2(3.9-8.9)$ & $7.98(4.46-12.5)$ & 0.45 \\
\hline \multicolumn{4}{|l|}{ Comorbidities } \\
\hline Cardiovascular disease, $\%$ & 85 & 66.6 & 0.38 \\
\hline Arterial hypertension, \% & 63.6 & 38.5 & \\
\hline Myocardial infarction, \% & 9.1 & 0 & \\
\hline Amyloidosis, \% & 5 & 0 & \\
\hline Diabetes, $\%$ & 15 & 23 & 0.67 \\
\hline \multicolumn{4}{|l|}{ Medications, \% } \\
\hline NSAIDs & 47.4 & 53.8 & \\
\hline \multicolumn{4}{|l|}{ RA medications } \\
\hline Methotrexate & 90 & & \\
\hline Other non-biologic DMARDs & 42.1 & & \\
\hline
\end{tabular}

$R A$ - rheumatoid arthritis; OA - osteoarthritis; BMI - body mass index; FM - total fat mass; FFM - fat-free mass; FVSC - visceral fat rating; ESR - erythrocyte sedimentation rate; CRP - C-reactive protein; HDL - high-density lipoprotein; $L D L$ - low-density lipoprotein; IL - interleukin; TNF - tumor necrosis factor; MIF - macrophage migration inhibitory factor; HGF - hepatocyte growth factor; DAS28 28-joint disease activity score in RA; NSAIDs - non-steroid anti-inflammatory drugs; DMARDs - disease-modifying anti-rheumatic drugs 
roid anti-inflammatory drug taking was also similar in both groups. However, there was a significant difference between RA and OA groups in body composition, which was normal in male patients with RA, but a more obese phenotype, accompanied by an excess of Fvsc (normal level =1-12), was characteristic for men with OA. As expected, in the group of RA patients there was an elevated erythrocyte sedimentation rate (ESR), and higher serum concentrations of $\mathrm{C}$-reactive protein (CRP) and pro-inflammatory cytokines (IL-6, MIF), compared with the OA group. The RA group included men with established and clinically active disease, treated mostly with disease-modifying drugs, and none received biological therapy.

Basal secretion of adipocytokines by SAAT explants obtained from RA and OA male patients is shown in Figure 1. In both tested groups SAAT produced spontaneously small and similar amounts of TNF and IL-10. The level of other adipocytokines' secretion was moderate

\section{A}

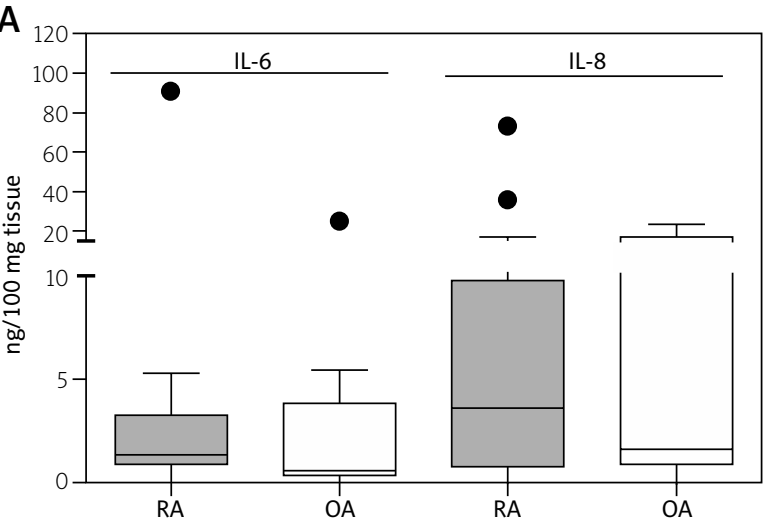

C

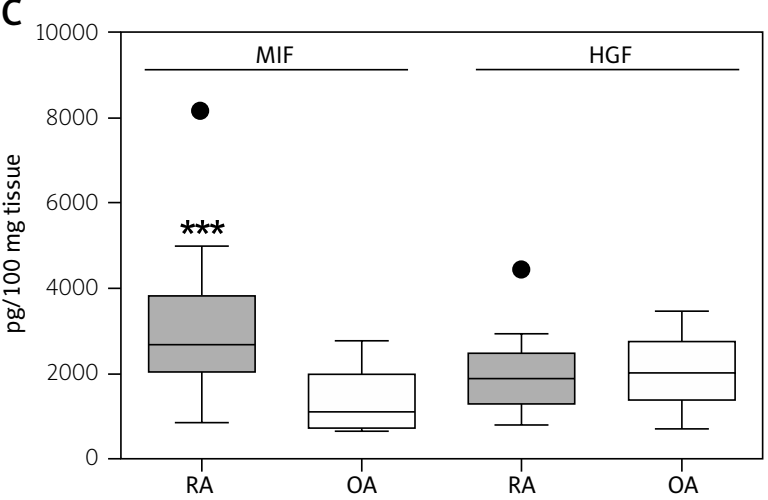

to large, and the only difference between patient groups was noted in the case of MIF and adiponectin, which were released in significantly higher amounts by SAAT of RA than OA patients. In both patient groups there was no significant correlation between serum concentrations and the amounts of SAAT-secreted adipocytokines, suggesting that this tissue is not the major source of circulating adipocytokines. The only exception was adiponectin in OA and IL- 6 in RA men, as serum concentrations and tissue release of these adipocytokines showed moderate to strong associations $(R=0.783, p=0.003$ and $R=0.49, p=0.03$, respectively) (data not shown).

In both patient groups stimulation of SAAT explants with pro-inflammatory IL-1 $\beta$ significantly up-regulated production of IL-6, IL-8, TNF, and IL-10 but failed to change secretion of MIF, HGF and classical adipokines (adiponectin, leptin) (Fig. 2). Although there was no significant difference in IL-1 $\beta$-triggered adipocytokine secretion by SAAT of RA and OA men, the release of at
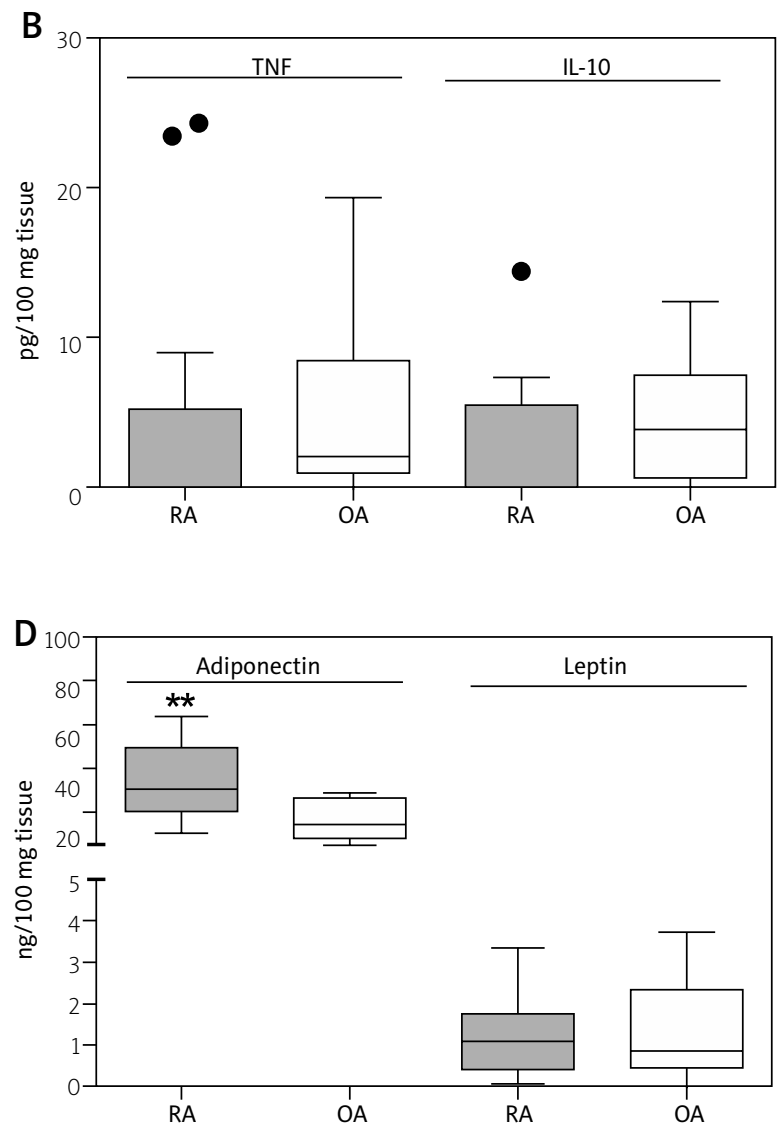

Fig. 1. Basal secretory activity of subcutaneous abdominal adipose tissue of rheumatoid arthritis (RA) and osteoarthritis $(\mathrm{OA})$ male patients. Tissue explants $(100 \mathrm{mg} / \mathrm{ml})$ obtained from $21 \mathrm{RA}$ and 13 OA men were cultured for $18 \mathrm{~h}$ and the concentrations of indicated adipocytokines in culture supernatants were measured by specific ELISAs. Boxes show the median and interquartile range (IQR), whiskers extend to $1.5 \times I Q R$ beyond the box, and outlier values are visualized as points. Statistically significant differences between RA vs. OA patients (asterisks) are indicated. ${ }^{* \star} p=0.01-0.001 ;{ }^{\star \star *} p=0.001-0.0001$. 

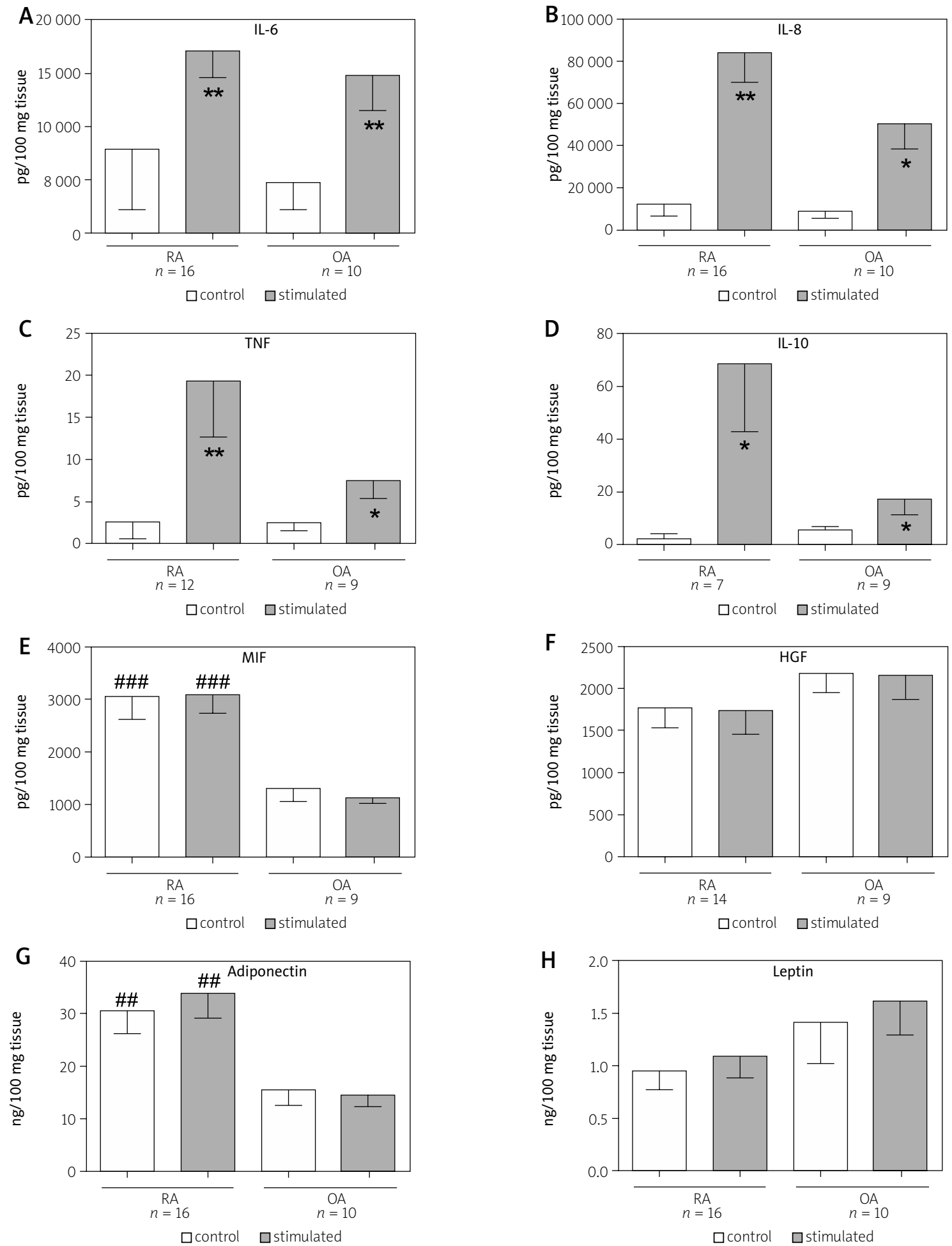

Fig. 2. Reactivity of subcutaneous abdominal adipose tissue of rheumatoid arthritis (RA) and osteoarthritis (OA) male patients to proinflammatory stimulus. Tissue explants $(100 \mathrm{mg} / \mathrm{ml})$ were cultured for $18 \mathrm{~h}$ in medium alone (control $=$ white bars) or were treated with IL-1 $\beta$ (stimulated = grey bars). Concentrations of indicated adipocytokines in culture supernatants were measured by specific ELISAs. Values are the mean and SEM of experiments performed using specimens from the indicated number of patients $(n)$. Statistically significant differences between stimulated and control cultures $\left({ }^{*} p=0.05-0.01 ;{ }^{* *} p=0.01-0.001\right)$ and RA

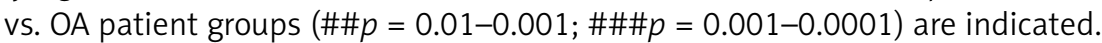


Table II. Relationship between serum and tissue originating adipocytokines and body composition in RA and OA male patients. Associations were assessed by Spearman's rank test, $R$ and $p$ values are shown

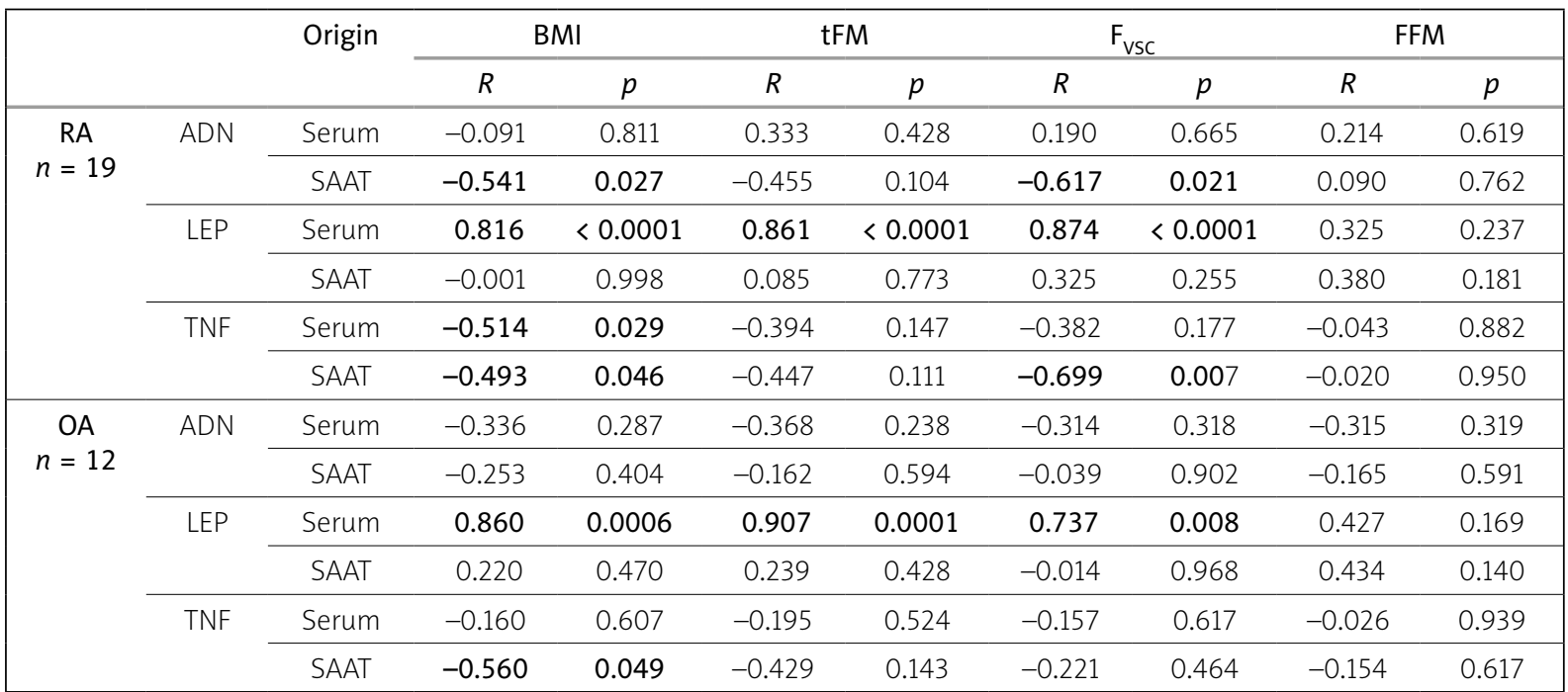

ADN - adiponectin; LEP - leptin; TNF - tumor necrosis factor; SAAT - subcutaneous abdominal adipose tissue; BMI - body mass index; $t F M$ - total fat mass; $F_{V S C}$ - visceral fat rating; FFM - fat-free mass

least TNF and IL-10 showed a tendency to be higher in RA patients.

As expected, in both groups of patients serum level of leptin showed a strong positive correlation with BMI, tFM and $F_{\text {vsc }}$ but leptin release from SAAT did not show such an association (Table II). In contrast, tissue-secreted (but not serum) adiponectin inversely and moderately correlated with $\mathrm{BMI}$ and $\mathrm{F}_{\mathrm{vsc}}$, although this relationship was found only in the RA male patient group. Interestingly, in both OA and RA groups SAAT-secreted TNF inversely correlated with $\mathrm{BMI}$ and in the latter group a similar correlation was also noted with $\mathrm{F}_{\mathrm{vsc}}$. In addition, in RA also serum TNF negatively correlated with BMI (Table II).

As shown in Figure 3, in RA patients classical adipokines secreted by SAAT, i.e. leptin and adiponectin, were positively and moderately correlated with disease duration and activity, respectively. In the OA group there was only a positive correlation between SAAT-released TNF and patients' age. We did not observe any significant correlations between tissue release of other adipokines and available demographic, clinical and laboratory data.

\section{Discussion}

In the present study higher concentrations of systemic inflammation markers (ESR, CRP) and pro-inflammatory cytokines (IL-6, MIF) were found in sera of RA than OA male patients (Table I). We recently reported similar differences between RA and OA female patients [14].
These observations are consistent with other findings recognizing $O A$ and RA as chronic inflammatory rheumatic diseases characterized by low- and high-systemic inflammation, respectively [1-5]. Accumulating evidence reveals an important role of adipocytokines in the regulation of the systemic inflammatory response and development of diseases related to contemporary civilization (insulin resistance, CVD), especially in obesity, where homeostasis of adipose tissue is shifted towards a more pro-inflammatory status $[9,17,18]$. It should be underlined that the contribution of white adipose tissue to this pathology depends on numerous factors, including gender, mass and anatomical location of fat depots as well as adipose tissue exposure to pro-inflammatory stimuli that modify composition of fat-resident immune cells, their activation state and pattern of secreted adipocytokines [11, 13-15, 18, 19]. It is well known that classical adipokines, i.e. leptin and adiponectin, exert opposite effects on food intake and energy homeostasis, which is reflected by the contrasting (positive for leptin and inverse for adiponectin) correlation of their circulating concentrations with fat mass and/or BMI [20, 21].

Our recently published data concerning female patients suffering from rheumatic diseases showed a more cachectic body composition but higher basal and IL-1 $\beta$ triggered secretory activity of SAAT in an RA than an OA cohort [14]. We concluded that these differences might result from stronger in vivo pre-exposure and higher reactivity of rheumatoid SAAT to pro-inflammatory challenge. Importantly, we also reported a significant correlation between SAAT-secreted adipokines and either body compo- 

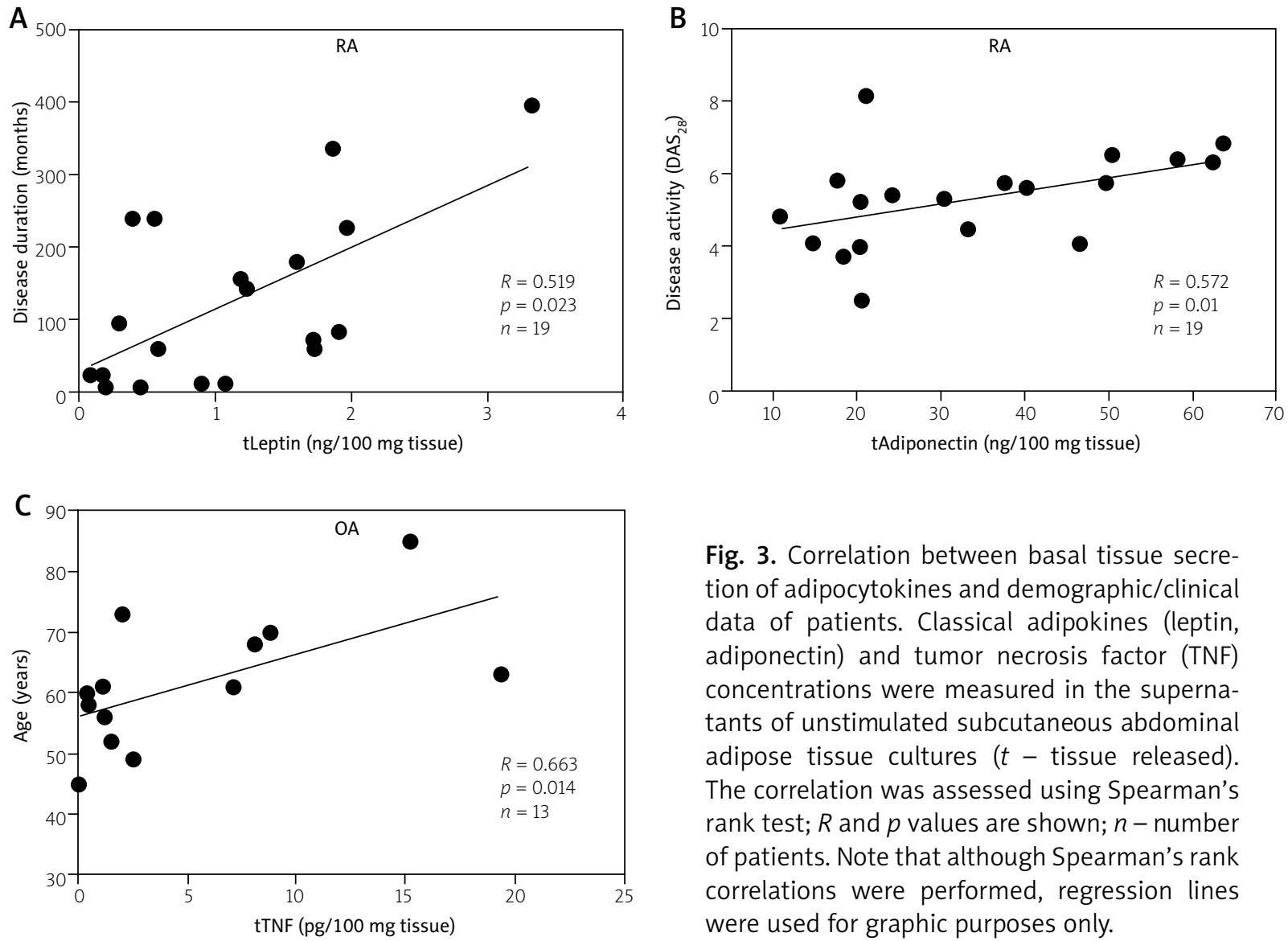

Fig. 3. Correlation between basal tissue secretion of adipocytokines and demographic/clinical data of patients. Classical adipokines (leptin, adiponectin) and tumor necrosis factor (TNF) concentrations were measured in the supernatants of unstimulated subcutaneous abdominal adipose tissue cultures ( $t$ - tissue released). The correlation was assessed using Spearman's rank test; $R$ and $p$ values are shown; $n$ - number of patients. Note that although Spearman's rank correlations were performed, regression lines were used for graphic purposes only.

sition (in RA women) or systemic inflammation markers (in OA women), suggesting a different clinical impact of secretory activity of this fat tissue. Consistently, in this female patient cohort we identified two independent

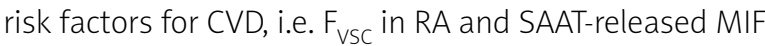
in OA, as well as SAAT-secreted HGF as an independent risk factor for amyloidosis in RA [14]. Unfortunately, due to the limited number of patients enrolled in the present study we were unable to confirm whether SAAT-secreted adipocytokines are independent risk factors for comorbidity in men affected with RA and OA.

The present results, focused on male patients, confirmed differences in body composition between OA and RA, i.e. lower content of FM and FFM and a lower visceral fat rating in the latter group (Table I). Interestingly, in RA two SAAT-released adipocytokines, i.e. adiponectin and TNF, showed an inverse correlation with BMI and $\mathrm{F}_{\mathrm{VSC}}$, while in $\mathrm{OA}$ there was negative correlation between SAAT-secreted TNF and BMI only (Table II). These observations suggest that in RA men adiponectin and TNF produced by SAAT may contribute to maintenance of normal body composition. This is in striking contrast to female RA patients where SAAT-derived adiponectin and TNF seem to have rather unwanted pro-cachectin

effects, because we previously found a negative correlation between secretion of these adipocytokines and the body contents of FFM [14]. Therefore, based on our previous and present results we conclude that adiponectin and TNF released by SAAT may control body composition in RA patients but the final effects of these adipocytokines depends on patients' gender. Consistently with the known role of leptin as a major regulator of body weight and composition [20], the present results demonstrate a positive correlation of its serum concentrations, but not SAAT-released leptin, with BMI, tFM and $F_{V s c}$ of male patients of both RA and OA groups (Table II). Similar associations between serum leptin concentrations and these indices were observed in female patients suffering from these rheumatic diseases [14]. However, in RA women also a positive correlation of SAAT-released leptin and BMI, tFM and FFM was found [14]. Taken together, our results indicate that in addition to the well-known association of the circulating leptin pool with body weight and composition (seen also in RA and OA patients independently of gender), SAAT-derived leptin may have similar importance in women with RA.

The striking dissimilarity of adipocytokine production by white adipose tissue from lean to obese individuals 
has been reported by others [18]. Therefore, the more obese phenotype of OA than RA men (Table I) suggested differences in the secretory activity of SAAT between these patient groups. Unexpectedly, we have only found higher basal release of adiponectin (which is consistent with a more lean phenotype) and MIF by SAAT of RA male patients compared with the OA group (Fig. 1). Our previous study showed similar differences between RA and OA female patients [14]. Migration inhibitory factor is a cytokine endowed with well-known pro-inflammatory, pro-atherogenic and chemokine-like activities. It also counteracts the immunosuppressive actions of glucocorticoids and is involved in the pathogenesis of many autoimmune and chronic inflammatory metabolic disorders $[22,23]$. This cytokine originates from both immune and non-immune cells, including adipocytes, and is produced by specific fat depots during a period of adipocyte expansion and fat inflammation [22]. On the other hand, MIF also induces muscle catabolism and contributes to the development of cancer-induced cachexia $[24,25]$. Because of these various functions it is hard to predict the net effect of high MIF secretion by SAAT to RA pathology. According to our previous [14] and present results, it is obvious that overproduction of MIF by SAAT is a characteristic feature of RA patients irrespective of gender. Interestingly, in both RA and OA male patients secretion of MIF (as well as classical adipokines and HGF) did not change upon stimulation of SAAT with pro-inflammatory IL-1 $\beta$ (Fig. 2), suggesting that in RA up-regulation of MIF is either a consequence of SAAT in vivo exposure to other unidentified factors or inherent in the disease.

Consistently with our former observations that IL-1 $\beta$ is a potent activator of adipose tissue from different anatomical locations and up-regulates a similar pattern of cytokines [11, 14, 19], in the present study we also noted up-regulation of pro-inflammatory (IL-6, IL-8, TNF) and anti-inflammatory (IL-10) cytokine secretion by IL-1 $\beta$ treated SAAT of RA and OA patients (Fig. 2). The reactivity of SAAT from both male patient groups to this stimulus was rather similar, which is in contrast to RA female patients characterized by higher IL-1 $\beta$-triggered secretion of IL- 6 and TNF by SAAT compared with OA women [14].

The positive correlation between SAAT-secreted adiponectin and RA clinical activity expressed by DAS28 observed in the present study (Fig. 3) is an interesting new finding. Adiponectin exists in globular and multimeric (low molecular weight trimers [LMW], middle molecular weight hexamers [MMW], and high-molecular weight oligomers [HMW]) forms, that differ in their biological activities [26, 27]. We have previously reported that in RA sera the HMW isoform predominates, while in synovial fluid similar proportions of HMW and LMW are present [28]. Importantly, we revealed that HMW/MMW adiponectin triggers pro-inflammatory and pro-destructive activities of rheumatoid fibroblast-like synoviocytes (FLS) and also predisposes these cells to a stronger response to IL-1 $\beta$. In addition, we found LMW adiponectin to incline rheumatoid FLS to a stronger response to IL-1 $\beta$ as well [28]. Altogether these findings point out pathogenic activity of adiponectin in RA that may explain its association with DAS28 observed in the present study. As for other SAAT-originated adipocytokines, the present results show a positive correlation between leptin and RA duration, as well as TNF and age of OA male patients (Fig. 3), indicating variability of SAAT secretory activity with RA progression or aging of OA men, respectively. It is worth noting that in female patients with rheumatic diseases the associations of SAAT-released adipocytokines with clinical and laboratory data were more complex, as in RA adiponectin inversely correlated with atherogenic index, while in OA leptin and HGF were positively and adiponectin negatively correlated with systemic inflammation markers [14].

\section{Conclusions}

In summary, this study shows higher basal secretion of adiponectin and MIF by SAAT of RA than OA male patients, characterized by high- and low-systemic inflammation, respectively. Moreover, we report that in RA and OA male patients secretory activity of this fat tissue shows different associations with demographic, clinical and laboratory data. In RA men SAAT-released adiponectin and TNF seem to contribute to maintenance of normal body composition and mass, and in addition tissue-originating adiponectin may play a pathogenic role as it positively correlates with disease activity, while secretion of leptin by SAAT increases with disease duration. In male patients with OA circulating, but not SAAT-originating, leptin is associated with obese phenotype (increased $\mathrm{BMI}, \mathrm{tFM}, \mathrm{F}_{\mathrm{vSC}}$ ), and release of TNF by SAAT increases with the patients' age. Based on our present and previous results [14], we conclude that differences in SAAT secretory activity and reactivity to inflammatory stimulation as well as in its possible clinical impact are smaller between RA and OA male than female patients.

\section{Acknowledgements}

We gratefully acknowledge all patients for their contribution to the study, and Iwona Janicka and Urszula Musiałowicz for outstanding technical assistance.

This study was funded by the National Institute of Geriatrics, Rheumatology and Rehabilitation (grant number S/16), Warsaw, Poland. The National Institute of Geriatrics, Rheumatology and Rehabilitation is sup- 
ported by a core grant from the Ministry of Science and Higher Education (Poland).

The authors declare no conflict of interest.

\section{References}

1. Kontny E. Pathogenesis of rheumatoid arthritis. Part I: Acquired immunity, genetic and environmental factors. Reumatologia 2011; 49: 47-54.

2. Kontny E. Pathogenesis of rheumatoid arthritis. Part II: Innate immunity, new therapeutic targets. Reumatologia 2011; 49: 115-121.

3. Kontny E. Pathogenesis of rheumatoid arthritis. Part III: Cytokines and joint destruction. Reumatologia 2011; 49: 180-186.

4. Cutolo M, Kitas GD, van Riel PL. Burden of disease in treated rheumatoid arthritis patients: going beyond the joint. Semin Arthritis Rheum 2014; 43: 479-488.

5. Berenbaum F. Osteoarthritis as an inflammatory disease (osteoarthritis is not osteoarthrosis). Osteoarthritis Cartilage 2013; 21: 16-21.

6. van Dijk GM, Veenhof C, Schellevis F, et al. Comorbidity, limitations in activities and pain in patients with osteoarthritis of the hip or knee. BMC Musculoskelet Disord 2008; 9: 95.

7. Johnson VL, Hunter DJ. The epidemiology of osteoarthritis. Best Pract Res Clin Rheumatol 2014; 28: 5-15.

8. Masuko K. Rheumatoid cachexia revised: a metabolic co-morbidity in rheumatoid arthritis. Front Nutr 2014; 1: 20.

9. Versini M, Jeandel PY, Rosenthal E, Shoenfeld Y. Obesity in autoimmune diseases: not a passive bystander. Autoimmun Rev 2014; 13: 981-1000.

10. Conde J, Scotece M, López V, et al. Adipokines: novel players in rheumatic diseases. Discov Med 2013; 15: 73-83.

11. Kontny E, Plebanczyk M, Lisowska B, et al. Comparison of rheumatoid articular adipose and synovial tissue reactivity to proinflammatory stimuli: contribution to adipocytokine network. Ann Rheum Dis 2012; 71: 262-267.

12. Eymard F, Pigenet A, Citadelle D, et al. Induction of an inflammatory and prodegenerative phenotype in autologous fibroblast-like synoviocytes by the infrapatellar fat pad from patients with knee osteoarthritis. Arthritis Rheum 2014; 66 2165-2174.

13. Lee MJ, Wu Y, Fried SK. Adipose tissue heterogeneity: implication of depot differences in adipose tissue for obesity complications. Mol Aspects Med 2013; 34: 1-11.

14. Kontny E, Zielińska A, Skalska U, et al. Distinct secretory activity and clinical impact of subcutaneous abdominal adipose tissue in women with rheumatoid arthritis and osteoarthritis. Inflammation 2016 (in press).

15. White UA, Tchoukalova YD. Sex dimorphism and depot differences in adipose tissue function. Biochim Biophys Acta 2014; 1842: 377-392.

16. Arnett FC, Edworthy SM, Bloch DA, et al. The American Rheumatism Association 1987 revised criteria for the classification of rheumatoid arthritis. Arthritis Rheum 1988; 31: 315-324.

17. Gustafson B. Adipose tissue, inflammation and atherosclerosis. J Atheroscler Thromb 2010; 17: 332-341.
18. Lee BC, Lee J. Cellular and molecular players in adipose tissue inflammation in the development of obesity-induced insulin resistance. Biochim Biophys Acta 2014; 1842: 446-462.

19. Kontny E, Prochorec-Sobieszek M. Articular adipose tissue resident macrophages in rheumatoid arthritis patients: potential contribution to local abnormalities. Rheumatology (Oxford) 2013; 52: 2158-2167.

20. Jéquier E. Leptin signalling, adiposity, and energy balance. Ann N Y Acad Sci 2002; 967: 379-388.

21. Dridi S, Taouis M. Adiponectin and energy homeostasis: consensus and controversy. J Nutr Biochem 2009; 20: 831-839.

22. Morrison MC, Kleemann R. Role of macrophage migration inhibitory factor in obesity, insulin resistance, type 2 diabetes, and associated hepatic co-morbidities: a comprehensive review of human and rodent studies. Front Immunol 2015; 6: 308.

23. Zernecke A, Bernhagen J, Weber C. Macrophage migration inhibitory factor in cardiovascular disease. Circulation 2008; 117: 1594-1602.

24. Benigni F, Atsumi T, Calandra T, et al. The proinflammatory mediator macrophage migration inhibitory factor induces glucose catabolism in muscle. J Clin Invest 2000; 106: 1291-1300.

25. Batista ML Jr, Neves RX, Peres SB, et al. Heterogenous time-dependent response of adipose tissue during the development of cancer cachexia. J Endocrinol 2012; 215: 363-373.

26. Scotece M, Conde J, López V, et al. Adiponectin and leptin: new targets in inflammation. Basic Clin Pharmacol Toxicol 2014; 114: 97-102.

27. Frommer KW, Schäffler A, Büchler C, et al. Adiponectin isoforms: a potential therapeutic target in rheumatoid arthritis? Ann Rheum Dis 2012; 71: 1724-1732.

28. Kontny E, Janicka I, Skalska U, Maśliński W. The effect of multimeric adiponectin isoforms and leptin on the function of rheumatoid fibroblast-like synoviocytes. Scand I Rheumatol 2015; 44: 363-368. 